\title{
EDITORIAL
}

\section{PRIMER CONGRESO ARGENTINO DE ANATOMÍA CLÍNICA}

\author{
Gustavo H. R. A. OTEGUI
}

Departamento de Anatomía, Facultad de Medicina, Universidad de Buenos Aires, Buenos Aires, Argentina.

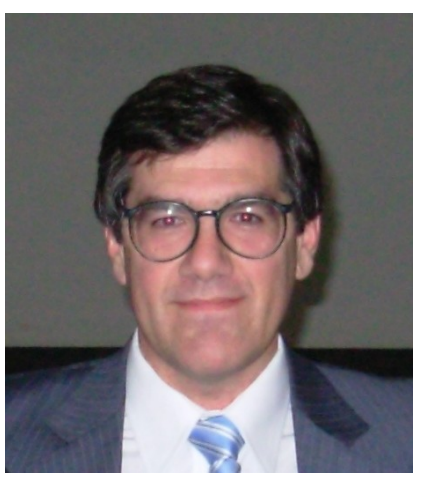

En la localidad de Huerta Grande, Córdoba, Argentina, del 23 al 26 de septiembre tuvo lugar el $1^{\circ}$ Congreso Argentino de Anatomía Clínica.

El marco geográfico no pudo ser mejor; el clima templado, la frescura y el cielo azul de sus amaneceres, la transparencia del aire de la noche coronada de estrellas y sus paredes de verdes arboledas y de ocres serranías.

La afabilidad de la gente del lugar, el entusiasmo con que fueron recibiendo y acogiendo a cada uno de los visitantes que llegaba, fue tallando el ambiente propicio para los días que vinieron.

El motivo del encuentro: la realización de un evento científico sin parangón, un congreso de anatomía clínica, el primero realizado en nuestro país.

La comisión organizadora estuvo presidida por la Profesora Dra. Susana N. Biasutto, acompañada por la Dra. Andrea V. Salas (secretaria) y el Dr. Marcos A. Spinelli (tesorero) y una pléyade de jóvenes entusiastas de la anatomía que se desvivían por hacer de este el mejor de los encuentros. Gracias a ellos todo estuvo en su lugar en el momento debido.
Para resaltar el impacto internacional que este evento ha tenido, baste resaltar de manera rápida el origen de los científicos extranjeros que asistieron al mismo: Australia, Austria, Brasil, Chile, Colombia, Ecuador, Estados Unidos de América, Italia, Perú, República Checa, Rumania, Suiza, Turquía y Uruguay.

El encuentro inició formalmente con palabras de la Presidente de la Asociación Argentina de Anatomía Clínica, quien dio una cálida bienvenida a los presentes e invitó a escuchar la conferencia inaugural del Profesor Emérito Dr. Stephen W. Carmichael (Mayo Clinic, Rochester, USA.), quien se desempeña actualmente como Editor en Jefe de la revista "Clinical Anatomy". El tema: "Integración longitudinal de la enseñanza de la anatomía a lo largo de la carrera de medicina". El Profesor Carmichael, señaló la importancia de articular de manera vertical los contenidos de la anatomía con la práctica médica y con las demás disciplinas de la carrera.

Al indicar la relevancia de la integración de la anatomía en la matriz del curriculum médico, se anticipaba de manera premonitoria, a lo que veríamos después, en las diferentes sesiones de este congreso de anatomía clínica.

Contacto a: Prof. Gustavo H. R. A. Otegui; Departamento de Anatomía, Facultad de Medicina, Universidad de Buenos Aires, Paraguay $21552^{\circ}$ piso (sector Uriburu), Ciudad Autónoma de Buenos Aires, Argentina; CP: C1121ABG; e-mail: gotegui@fmed.uba.ar 
El desarrollo del evento avanzó a través de: conferencias plenarias a cargo de especialista que reseñaron en apretado resumen las principales corrientes del pensamiento anatómico contemporáneo; mesas redondas y paneles, en las que pudimos apreciar de cada tema, las distintas aristas, finamente esculpidas por cada uno de los expertos que expusieron. $Y$ no faltaron las sesiones de debate, centradas en diferentes tópicos de la anatomía, la investigación, la docencia en el pregrado y en el posgrado.

Y finalmente, en esta importante reunión, se dieron cita, más de un centenar de presentaciones de trabajos científicos, previamente evaluados por el comité de referato constituido ad hoc por pares evaluadores. Las modalidades de presentación fueron las habituales láminas (posters) y comunicaciones orales.

Llegados al día 26, todos comprendimos que, discretamente las horas habían pasado, y sin notarlo, cada uno de nosotros, venidos de diferentes lugares, nos habíamos vuelto parte de una gran familia internacional. A no dudarlo, Huerta Grande, fue en esos días el lugar de un congreso, y la mejor excusa para aprender lo último y lo nuevo de la anatomía, rodeado de jóvenes y viejos amigos, que sienten la misma pasión que nosotros por el tema.

Con la vitalidad y fortaleza obtenida en este encuentro, preparemos el próximo, con la esperanza de volver a vernos en Tucumán en 2011. 